\title{
BLACK HOLES AND QUASIBLACK HOLES IN EINSTEIN-MAXWELL THEORY
}

\author{
REINHARD MEINEL, MARTIN BREITHAUPT and YU-CHUN LIU \\ University of Jena, Theoretisch-Physikalisches Institut, \\ Max-Wien-Platz 1, 07743 Jena, Germany \\ meinel@tpi.uni-jena.de
}

\begin{abstract}
Continuous sequences of asymptotically flat solutions to the Einstein-Maxwell equations describing regular equilibrium configurations of ordinary matter can reach a black hole limit. For a distant observer, the spacetime becomes more and more indistinguishable from the metric of an extreme Kerr-Newman black hole outside the horizon when approaching the limit. From an internal perspective, a still regular but non-asymptotically flat spacetime with the extreme Kerr-Newman near-horizon geometry at spatial infinity forms at the limit. Interesting special cases are sequences of Papapetrou-Majumdar distributions of electrically counterpoised dust leading to extreme Reissner-Nordström black holes and sequences of rotating uncharged fluid bodies leading to extreme Kerr black holes.
\end{abstract}

\section{Introduction}

Spherically symmetric (uncharged) perfect fluid bodies in equilibrium cannot reach a black hole limit parametrically. Buchdahl has shown ${ }^{1}$ that the Schwarzschild coordinate radius of such a fluid ball is always greater than (9/8) $2 M$, where $M$ denotes the gravitational mas 3 . Correspondingly, the relative gravitational redshift $z$ of photons emitted from the fluid's surface and received at infinity is bounded by $z<2$.

With rotation and/or electric charge a (quasi) black hole limit of regular equilibrium configurations is possible. In this parameter limit, if discussed from an external perspective, the spacetime of an extreme Kerr-Newman black hole outside the horizon forms. From an internal perspective, a still regular but non-asymptotically flat spacetime with the extreme Kerr-Newman near-horizon geometry at spatial infinity results. The first example was given with the extreme relativistic limit of a uniformly

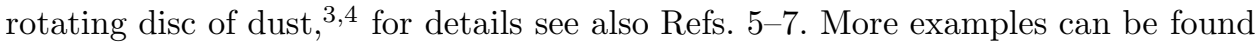
in Refs. 8-13. The simplest way to construct such "quasiblack holes" is to use the parametric compression of static configurations of "electrically counterpoised dust (ECD)", also called "Bonnor stars" 1420

In the following, we will touch on some general aspects of the black hole limit in the two special cases: (i) perfect fluid configurations (angular momentum but no charge) leading to extreme Kerr black holes and (ii) ECD configurations (electric charge but no angular momentum) leading to extreme Reissner-Nordström black holes. We conclude with some comments on rotating discs of charged dust. This model provides a continuous connection between the two cases mentioned above.

${ }^{a}$ We use units in which $G=c=1$.

${ }^{\mathrm{b}}$ For a constructive proof of the Kerr-Newman black hole uniqueness including the extreme case, see Ref. 2 


\section{Black hole limit of rotating fluid bodies in equilibrium}

A black hole limit of a stationary and axisymmetric, uniformly rotating perfect fluid body occurs if and only if $M-2 \Omega J \rightarrow 0$ holds at the limit, 21 where $\Omega$ and $J$ denote angular velocity and angular momentum. Consequently, from an external perspective, the spacetime of an extreme Kerr black hole outside the horizon forms. A rigorous proof for the existence of such a limit was given with the analytic solution to the disc of dust problem. ${ }^{4 / 5}$ Convincing numerical evidence for such a limit was also found for fluid ring solutions with various equations of state $\frac{10}{12}$

\section{Black hole limit of electrically counterpoised dust configurations}

The black hole limit of ECD configurations can be treated in quite a general manner, by applying a simple scaling transformation to any asymptotically flat PapapetrouMajumdar solution of the Einstein-Maxwell equations, corresponding to a localized ECD distribution. ${ }^{20}$ It is interesting to note that although the "outer world" at the limit is always given by the spherically symmetric extreme Reissner-Nordström metric outside the horizon, the "inner world" does not need to show any spatial symmetry in general.

\section{Rotating discs of charged dust}

The problem defining the global solution to the Einstein-Maxwell equations corresponding to a uniformly rotating (infinitesimally thin) disc of electrically charged dust with constant specific charge $\epsilon(0 \leq \epsilon \leq 1$, we are using Gauss units) can be formulated as a boundary value problem for the Ernst equations ${ }^{22}$

$$
\begin{gathered}
f \Delta \mathcal{E}=(\nabla \mathcal{E}+2 \bar{\Phi} \nabla \Phi) \cdot \nabla \mathcal{E}, \quad f \Delta \Phi=(\nabla \mathcal{E}+2 \bar{\Phi} \nabla \Phi) \cdot \nabla \Phi \\
\text { with } \quad f \equiv \Re \mathcal{E}+|\Phi|^{2}, \quad \Delta=\frac{\partial^{2}}{\partial \rho^{2}}+\frac{1}{\rho} \frac{\partial}{\partial \rho}+\frac{\partial^{2}}{\partial \zeta^{2}}, \quad \nabla=\left(\frac{\partial}{\partial \rho}, \frac{\partial}{\partial \zeta}\right) .
\end{gathered}
$$

The boundary conditions on the disc $\left(\zeta=0,0 \leq \rho \leq \rho_{0}\right)$ take a simple form in the corotating frame of reference (indicated by primes):

$$
\rho_{0} \frac{\partial}{\partial \rho}\left(\sqrt{f^{\prime}}+\epsilon \Re \Phi^{\prime}\right)=0, \rho_{0} \frac{\partial}{\partial \zeta}\left(\Re \Phi^{\prime}+\epsilon \sqrt{f^{\prime}}\right)=0, \Im \mathcal{E}^{\prime}=0, \Im \Phi^{\prime}=0 .
$$

They have to be complemented by the asymptotic flatness conditions $\mathcal{E} \rightarrow 1, \Phi \rightarrow 0$ as $\rho^{2}+\zeta^{2} \rightarrow \infty$ and a regularity condition at the rim of the disc. Except from a scaling parameter $\left(\rho_{0}\right.$ or $\left.M\right)$, the solution can be discussed in terms of its dependence on the two parameters $\epsilon$ and $\gamma \equiv 1-\sqrt{f(\rho=0, \zeta=0)}$ with $0<\gamma \leq 1$. The limit $\epsilon \rightarrow 0$ leads back to the disc of dust solution discussed above. For $\epsilon \rightarrow 1$, one obtains a special (non-rotating) ECD configuration. The Newtonian limit is characterized by $\gamma \ll 1$, whereas $\gamma \rightarrow 1$ is expected to lead to the black hole limit. The gravitational mass of the disc can, in general, be expressed as

$$
M=2 \Omega J+\left(1-\gamma+\epsilon \alpha_{0}\right) M_{0},
$$


where $\alpha_{0} \equiv \Re \Phi(\rho=0, \zeta=0)$ and $M_{0}$ denotes the baryonic mass. Accordingly, the electric charge of the disc is given by $Q=\epsilon M_{0}$. The factor $1-\gamma+\epsilon \alpha_{0}$ on the righthand side of Eq. (2) is equal to the constant value of $\sqrt{f^{\prime}}+\epsilon \Re \Phi^{\prime}$ on the disc, cf. the first boundary condition in (11). At the limit $\gamma \rightarrow 1$, we expect to obtain $f^{\prime}=0$ and thus $\Re \Phi^{\prime}=$ constant on the disc. Denoting this constant by $\phi\left(\phi=\alpha_{0}\right)$, the relation (2) reduces to $M=2 \Omega J+\phi Q$, which looks like the Smarr formula ${ }^{23}$ for an extreme Kerr-Newman black hole. For finite $M$, the coordinate radius $\rho_{0}$ is expected to shrink to zero at the limit. Indeed, the horizon of an extreme Kerr-Newman black hole is located at $\rho=0, \zeta=0$ in the coordinates used here. Moreover, the KerrNewman solution satisfies the conditions $f^{\prime}=0, \Re \Phi^{\prime}=$ constant, $\Im \mathcal{E}^{\prime}=0$ and $\Im \Phi^{\prime}=0$ on the horizon.

A post-Newtonian expansion of the disc of charged dust solution will be published elsewhere ${ }^{\sqrt[24]{1}}$ It remains a challenge to find an explicit analytic solution as is possible for the uncharged case $(\epsilon=0)$.

\section{Acknowledgments}

This research was supported by the Deutsche Forschungsgemeinschaft (DFG) through the Graduiertenkolleg 1523 "Quantum and Gravitational Fields".

\section{References}

1. H. A. Buchdahl, Phys. Rev. 116, 1027 (1959).

2. R. Meinel, Class. Quantum Grav. 29, 035004 (2012).

3. J. M. Bardeen and R. V. Wagoner, Astrophys. J. 167, 359 (1971).

4. G. Neugebauer and R. Meinel, Phys. Rev. Lett. 75, 3046 (1995).

5. R. Meinel, Ann. Phys. (Leipzig) 11, 509 (2002).

6. R. Meinel, M. Ansorg, A. Kleinwächter, G. Neugebauer and D. Petroff, Relativistic Figures of Equilibrium (Cambridge University Press, Cambridge, 2008).

7. A. Kleinwächter, H. Labranche and R. Meinel, Gen. Rel. Grav. 43, 1469 (2011).

8. P. Breitenlohner, P. Forgács and D. Maison, Nucl. Phys. B 442, 126 (1995).

9. A. Lue and E. J. Weinberg, Phys. Rev. D 61, 124003 (2000).

10. M. Ansorg, A. Kleinwächter and R. Meinel, Astrophys. J. 582, L87 (2003).

11. T. Fischer, S. Horatschek and M. Ansorg, Mon. Not. R. Astron. Soc. 364, 943 (2005).

12. H. Labranche, D. Petroff and M. Ansorg, Gen. Rel. Grav. 39, 129 (2007).

13. J. P. S. Lemos and V. T. Zanchin, Phys. Rev. D 81, 124016 (2010).

14. W. B. Bonnor and S. B. P. Wickramasuriya, Mon. Not. R. Astron. Soc. 170, 643 (1975).

15. W. B. Bonnor, Class. Quantum Grav. 15, 351 (1998).

16. W. B. Bonnor, Class. Quantum Grav. 16, 4125 (1999).

17. J. P. S. Lemos and E. J. Weinberg, Phys. Rev. D 69, 104004 (2004).

18. J. P. S. Lemos and O. B. Zaslavskii, Phys. Rev. D 76, 084030 (2007).

19. W. B. Bonnor, Gen. Rel. Grav. 42, 1825 (2010).

20. R. Meinel and M. Hütten, Class. Quantum Grav. 28, 225010 (2011).

21. R. Meinel, Class. Quantum Grav. 23, 1359 (2006).

22. F. J. Ernst, Phys. Rev. 168, 1415 (1968).

23. L. Smarr, Phys. Rev. Lett. 30, 71 (1973).

24. S. Palenta and R. Meinel, in preparation. 